\title{
Comparison of Treatment Results and Satisfaction Levels of Endoscopic Thoracic Sympathectomy Techniques Performed in Primary Focal Hyperhidrosis
}

(1) Servet Ozdemir

University of Health Sciences Turkey, Bakirkoy Dr. Sadi Konuk Education and Research Hospital, Clinic of Thoracic Surgery, Istanbul, Turkey

\section{Abstract}

\begin{abstract}
Aim: This study aims to investigate whether the cauterization or clipping techniques performed in endoscopic thoracic sympathectomy surgery (ETS) in a tertiary center differ in terms of postoperative results and satisfaction level and to compare the results according to the surgical methods performed.

Methods: In the study, the data of 94 cases treated with ETS due to primary focal hyperhidrosis in a thoracic surgery clinic between 2015 and 2018, were retrospectively analyzed. Patients consisted of two different surgical groups. The cauterization method was performed on $56(59.6 \%)$ of the patients whereas the clipping was performed on 38 (40.4\%) of them. The patients were compared in terms of recurrence, reflex sweating, and satisfaction, as well as demographic characteristics.
\end{abstract}

Results: As a result of the Univariate Logistic Regression Analysis, it was found that the increase in age among all cases increased the risk of dissatisfaction with the treatment [odds ratio (OR): 1.11, 95\% confidence interval $(\mathrm{Cl}): 1.03-1.20, p=0.005$ ] whereas the cauterization method (OR: $0.26,95 \% \mathrm{Cl}: 0.09-0.77, \mathrm{p}=0.015)$, absence of recurrence (OR: $0.03,95 \% \mathrm{Cl}: 0.01-0.12, \mathrm{p}<0.001)$, and absence of reflex sweating (OR: $0.09,95 \% \mathrm{Cl}: 0.01-0.68, \mathrm{p}=0.020$ ) increased the satisfaction in all patients.

Conclusion: The increase in age, the clipping method, the presence of recurrence, and the presence of reflexive sweating are the risks of decreasing the level of patient satisfaction.

Keywords: Primary local hyperhidrosis, endoscopic thoracic sympathectomy, sympathectomy, endoscopic sympathetic clips

\section{Introduction}

Hyperhidrosis is defined as a pathological dimension of sweating more than physiologically needed to control body temperature (1). Hyperhidrosis, which is seen in approximately $3 \%$ of the general population, is frequently detected in people between the ages of 25 and 64, and it creates a psychological and social burden as it affects the daily activities of people negatively (2). Although there is no standard algorithm in the treatment of hyperhidrosis, it can be examined into two main groups as conservative medical methods (topical treatments, systemic treatments, Iontophoresis and botulinum toxin injection) and interventional treatment methods (local surgical procedures, stereotactic percutaneous thermal ablation, and sympathectomy) $(3,4)$.

In the treatment of hyperhidrosis, it has been stated that ETS is the best surgical method although the superiority of the methods (cauterization or clip) used to interrupt the sympathetic chain is controversial, and the success of the performed surgical method may vary depending on the age of the patient, the severity of the disease, the type of the disease, and the area where the surgical procedure was performed $(T 2, T 3, T 4)(1,5)$. It has been also mentioned that there is a significant increase in the quality of life of patients with hyperhidrosis after the surgical procedure and that those people have a high degree of satisfaction

Address for Correspondence/Yazışma Adresi: Servet Ozdemir, University of Health Sciences Turkey, Bakırkoy Dr. Sadi Konuk Education and Research Hospital, Clinic of Thoracic Surgery, Istanbul, Turkey Phone: +90 2124147171 E-mail: servetozdemir74@hotmail.com ORCID: orcid.org/0000-0002-8458-0772 Received/Geliş Tarihi: 20.11.2020 Accepted/Kabul Tarihi: 19.01.2021 
with the treatment. The surgical method is, therefore, the most appropriate option as the primary-care in those cases (6). In a study in which the efficacy of cauterization and clipping methods were compared in the treatment, both two methods were found quite effective in the treatment of primary focal hyperhidrosis, although the recurrence rate of the disease was higher in the cauterization group (7).

We aimed to investigate whether the cauterization or clipping techniques performed in endoscopic thoracic sympathectomy surgery (ETS) in a tertiary center differ in terms of postoperative results and satisfaction level and to compare the results according to the surgical methods performed.

\section{Methods}

The data of one hundred fifteen cases treated with ETS in the thoracic surgery clinic due to the primary focal hyperhidrosis (palmar and/or Axillary) were retrospectively analyzed between January 1, 2015, and December 31, 2018, in the study. Ethical consent for the study was obtained from the ethics committee of the hospital before the study (protocol number: 2020/479). Cases operated at least two years ago were evaluated in the study. The contact information of the patients and their relatives was obtained from the medical records, and patients were gone through the phone in order to learn their satisfaction level with the treatment. Since 21 patients could not be contacted in the study, the data of the remaining 94 cases were examined. Patients consisted of two different surgical groups. The cauterization method was performed on 56 (59.6\%) of the patients whereas the clipping was performed on $38(40.4 \%)$ of them. The patients were compared in terms of recurrence, reflex sweating, and satisfaction, as well as demographic characteristics. The cases who were contacted via phone were informed about the study, the consent was obtained from the patients that they volunteered to participate in the study, and their treatment satisfaction was questioned. In these interviews, each patient was asked to evaluate how satisfied they were with the hyperhidrosis treatment in a standard way, as not satisfied (1), partially satisfied (2), moderately satisfied (3), satisfied (4), and very satisfied (5). The reason why this method was preferred in the research was the absence of a valid and reliable measurement tool that measures treatment satisfaction to question via the phone in the country. Then, the responses of patients who underwent surgery with a diagnosis of hyperhidrosis in the past were recorded on the case form. Besides, demographic and clinical characteristics of the patients such as age, education level, length of stay, and the type of surgical procedures were registered in the case form.
The inclusion criteria for the study were the diagnosis of primary focal hyperhidrosis in patients who did not benefit from any medical treatments performed before the ETS and the patient's voluntary participation in the study. Patients who were not contacting by phone after surgery $(n=21)$ were excluded.

\section{Surgery Procedure}

Based on the examination of medical records, it was observed that all of the patients were intubated with a double-lumen endotracheal tube under general anesthesia, and bilateral ETS surgery was performed in the same session. Besides, the patients were operated on in a semi-sitting position and by providing single-lung ventilation. Approximately $1-1.5 \mathrm{~cm}$ single-port incision was made in the fourth intercostal space in the midaxillary line. Moreover, zero-degree, $5 \mathrm{~mm}$ optical, and endoscopic instruments were placed in the thorax. With the determination of thoracic sympathetic chain in cases, "T3-T4" sympathetic ganglion and chain intervention (clip or hook electrocautery) were performed as standard in 2 levels in all patients. Subsequently, the nerve of Kuntz was also cauterized in all patients. It was determined that care was taken not to damage the stellate ganglion and intercostal vessels located near the surgical site and after the hemorrhage and air leak control, one Nelaton catheter was inserted into the thorax from the same incision site. To ensure lung expansion, the lungs were ventilated so that the other end of the catheter was in a container with serum physiological. Then, when it was observed that the air outlet from the tip of the catheter had completely ceased, the catheter was withdrawn and the incision was closed without placing the thorax tube. The procedure was performed for the other side in the same session.

\section{Statistical Analysis}

The clinical and demographic characteristics of the cases diagnosed with primary focal hyperhidrosis were evaluated by descriptive statistical analyzes such as number, percentage, mean, and standard deviation. Numerical data such as age and length of stay among the cases with the cauterization and the clipping were obtained using independent groups t-test whereas proportional data such as gender, recurrence, and treatment satisfaction level were evaluated by chi-square analyzes (Fischer's exact chi-square analysis was used if the proportional data was below 5\%). Numerical data such as age and length of stay between cases with and without recurrence were examined using the Mann-Whitney $U$ test whereas proportional data such as gender and type of surgical procedure was evaluated by chi-square analysis (Fischer's exact chi-square analysis was used if the proportional data was below 5\%). Satisfaction with the treatment was 
divided into two groups: insufficient (dissatisfied, partially satisfied) and sufficient (moderate, good, and very good satisfied). Therefore, univariate logistic regression analysis was used to examine the probability of dissatisfaction with the treatment at a sufficient level and the variables that may have an impact on the probability of recurrence. The significance level for all analyzes was set as $p<0.05$. IBM SPSS 22.0 program was used to evaluate the analyzes.

\section{Results}

The mean age of the cases evaluated in the study was $22.82 \pm 6.88$ years [minimum ( $\mathrm{min}$ ): 12.00 -maximum ( $\max$ ): $44.00]$, and $44(46.2 \%)$ of the patients were male. In the evaluation made after the operation, it was found that the disease recurred in $14(14.9 \%)$ of all cases and reflex sweating was found in $62(66.0 \%)$ of the cases. The mean length of stay was $2.26 \pm 1.01$ days (min: 1 -max: 5). It was evaluated that complication developed in $3(3.2 \%)$ cases (ptosis in 1 patient, ongoing inadequate expansion in 2 patients). It was detected that 2 patients with inadequate expansion were treated with tube thoracostomy. In the interview about satisfaction with treatment after the operation, $12(12.8 \%)$ of the cases were reported as 1 (not satisfied) whereas $6(6.4 \%)$ of them reported the satisfaction level as 2 (partially satisfied), 11 (11.7\%) of them reported as 3 (moderately satisfied), 21 (22.3\%) of them reported 4 (satisfied), and $44(46.8 \%)$ of them reported as 5 (very satisfied).

According to the independent groups t-test, the mean of age $(t=-1.35, p=0.182)$, the mean of length of stay $(t=-0.48, p=0.633)$, and the mean of follow-up years $(t=0.11, p=0.108)$ were found to be statistically similar. In comparison between surgical methods according to chisquare analysis, gender $\left(X^{2}=0.26, p=0.609\right)$, recurrence $\left(X^{2}=0.63, p=0.429\right)$, reflex sweating $\left(X^{2}=0.00, p=0.977\right)$, and complication $\left(X^{2}=0.07, p=0.999\right)$ rates were not found to be statistically significantly different. Based on the Fischer's exact chi-square analysis, it was found that satisfaction rates were not also statistically significantly different $\left(X^{2}=7.28, p=0.114\right)$ between surgical procedures performed (Table 1). Besides, the pairwise comparison of the groups was examined using the Bonferroni correction and it was found that there was no statistically significant difference $(p>0.05)$ between the groups (Graphic 1).

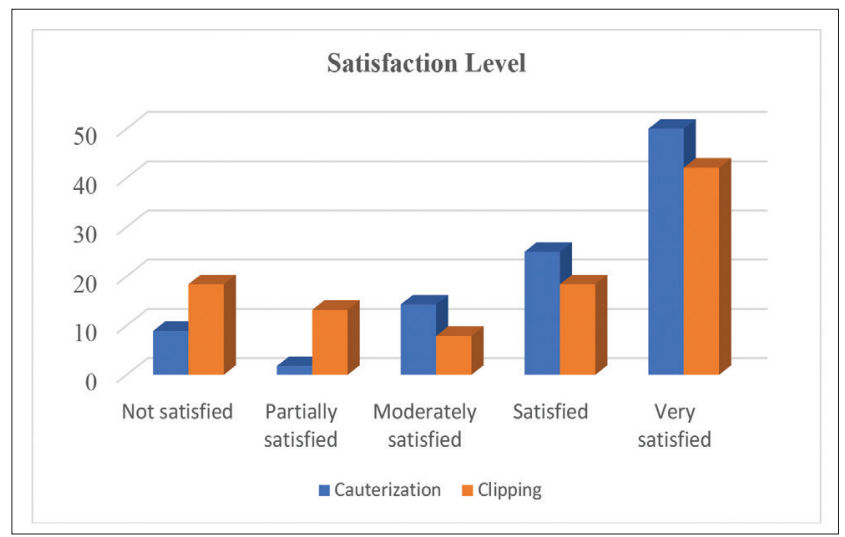

Graphic 1. Comparison of the satisfaction levels of the patients according to the surgical method

\begin{tabular}{|c|c|c|c|c|c|}
\hline & & Cauterization & Clipping & $\mathbf{X}^{2} / \mathbf{t}$ & $\mathrm{p}$ \\
\hline \multicolumn{2}{|l|}{ Age (Year) (Mean \pm SD) } & $22.04 \pm 6.10$ & $23.97 \pm 7.84$ & -1.35 & 0.182 \\
\hline \multirow{2}{*}{ Gender (n/\%) } & Male & $25(44.6)$ & $19(50.0)$ & 0.26 & 0.609 \\
\hline & Female & $31(55.4)$ & $19(50.0)$ & & \\
\hline \multicolumn{2}{|c|}{ Length of follow-up (year) (Mean \pm SD) } & $2.98 \pm 0.96$ & $3.29 \pm 0.80$ & 0.011 & 0.108 \\
\hline \multicolumn{2}{|c|}{ Length of Stay (day) (Mean \pm SD) } & $2.21 \pm 0.91$ & $2.32 \pm 1.14$ & -0.48 & 0.633 \\
\hline \multirow{2}{*}{ Recurrence (n/\%) } & No & 49 (87.5\%) & $31(81.6)$ & 0.63 & 0.429 \\
\hline & Yes & $7(12.5)$ & $7(18.4)$ & & \\
\hline \multirow{2}{*}{ Reflex sweating (n/\%) } & No & $19(33.9)$ & $13(34.2)$ & 0.00 & 0.977 \\
\hline & Yes & $37(66.1)$ & $25(65.8)$ & & \\
\hline \multirow{2}{*}{ Complication (n/\%) } & No & $54(96.4)$ & $37(97.4)$ & 0.07 & 0.999 \\
\hline & Yes & $2(3.6)$ & $1(2.6)$ & & \\
\hline \multirow{5}{*}{ Satisfaction (n/\%) } & Not satisfied (1) & $5(8.9)$ & $7(18.4)$ & 7.28 & $0.114^{a}$ \\
\hline & Partially satisfied (2) & $1(1.8)$ & $5(13.2)$ & & \\
\hline & Moderately satisfied (3) & $8(14.3)$ & $3(7.9)$ & & \\
\hline & Satisfied (4) & $14(25.0)$ & $7(18.4)$ & & \\
\hline & Very satisfied (5) & $28(50.0)$ & $16(42.1)$ & & \\
\hline
\end{tabular}

SD: Standard deviation, $\mathrm{X}^{2}$ : Chi-square analysis, t: Independent groups t-test, a: Fischer's exact, chi-square analysis 
The insufficient level of satisfaction with treatment $(n=18 ; 18.1 \%)$ was coded as negative in regression analysis, whereas the sufficient level of satisfaction with treatment ( $n=78 ; 81.9 \%$ ) was coded as positive in the study. According to the results of univariate logistic regression analysis, increment in age [odds ratio (OR): 1.11, confidence interval $(\mathrm{Cl}): 1.03-1.20 ; p=0.005]$ was found to increase the probability of dissatisfaction with treatment. Besides, the cauterization method (OR: 0.26, $\mathrm{Cl}$ : 0.09-0.77; $p=0.015)$, the absence of recurrence (OR: $0.03, \mathrm{Cl}: 0.01-0.12 ; p<0.001)$, and the absence of reflex sweating (OR: 0.09, Cl: 0.01-0.68; $p=0.020$ ) was found to reduce the probability of dissatisfaction with the treatment (Table 2).

Of the 14 cases with post-treatment recurrence of hyperhidrosis, 5 (35.7\%) were male, 7 (50\%) were cauterized, $13(92.9 \%)$ had reflex sweating whereas the mean age of these cases was 24.93 years (median: 23.50, min: 15.00-max: 43.00) and the mean of the length of stay was 2.57 days (median: 2.50, min: 1.00-max: 4.00). Besides, of the 80 cases without recurrence, 39 (48.8\%) were male, 49 (61.3\%) were cauterized, 49 (61.3\%) had reflex sweating, and $3(3.8 \%)$ had complications, whereas the mean age of these cases was 22.45 (median: 20.00) and the mean length of stay was 2.20 (median=2.00). Based on the chi-square analysis and Mann-Whitney $U$ tests, it was found that the other variables were not statistically different between the cases with and without recurrence, except for the reflex sweating rate $\left(X^{2}=5.30\right.$, $\mathrm{p}=0.030$ ).

\begin{tabular}{|l|l|l|l|}
\hline $\begin{array}{l}\text { Table 2. Univariable logistic } \\
\text { associated with treatment dissatisfaction }\end{array}$ & \multicolumn{1}{l}{ regression analysis result } \\
\hline & OR & $\mathbf{9 5 \%} \mathbf{~ C l}$ & p \\
\hline Age & 1.11 & $1.03-1.20$ & 0.005 \\
\hline Gender (Male) & 0.67 & $0.24-1.92$ & 0.456 \\
\hline Surgical method (cauterization) & 0.26 & $0.09-0.77$ & 0.015 \\
\hline Recurrence (No) & 0.03 & $0.01-0.12$ & $<0.001$ \\
\hline Reflex sweating (No) & 0.09 & $0.01-0.68$ & 0.020 \\
\hline Length of stay & 0.83 & $0.47-1.42$ & 0.497 \\
\hline OR: Odds ratio, Cl: Confidence interval & & & \\
\hline
\end{tabular}

\begin{tabular}{|c|c|c|c|}
\hline & OR & $95 \% \mathrm{Cl}$ & $\mathbf{p}$ \\
\hline Age & 1.05 & $0.97-1.13$ & 0.218 \\
\hline Gender (Male) & 0.58 & $0.18-1.90$ & 0.371 \\
\hline Surgical method (cauterization) & 0.63 & $0.20-1.98$ & 0.431 \\
\hline Length of stay & 1.43 & $0.82-2.47$ & 0.205 \\
\hline \multicolumn{4}{|l|}{ OR: Odds ratio, Cl: Confidence interval } \\
\hline
\end{tabular}

Recurrence $(n=14)$ was coded as negative in regression analysis, and non-recurrence $(n=80)$ was coded as positive in the study. Additively, multiple analysis was not preferred due to the limited distribution of the number of subjects in terms of the variables. Regarding the results of univariate logistic regression analysis, age, gender, surgical method, and length of stay were not statistically effective factors $(p>0.05)$ on the probability of increasing recurrence in hyperhidrosis cases (Table 3).

\section{Discussion}

Satisfaction rates of patients in the study were found to be similar among the cases diagnosed with primary focal hyperhidrosis, in which cauterization or clipping method was performed in the past. Moreover, the recurrence rates were identified as statistically similar between the two groups. As a result of the logistic regression analysis, it was determined that the increment in age among all cases increased the probability of dissatisfaction with the treatment whereas the method of cauterization, the absence of recurrence, and the absence of reflex sweating were found to reduce the probability of dissatisfaction with the treatment.

In the literature, different ETS methods (cauterization, clipping) performed in cases diagnosed with primary focal hyperhidrosis have been found to differ in terms of treatment success and patient satisfaction $(1,5)$. Findikcioglu et al. (7) stated that the clipping method is more successful than other methods in increasing the quality of life of patients. In a meta-analysis study, it was stated that although the clipping method is more successful than cauterization in terms of patient satisfaction and recurrence rate, this method may have a higher risk for complications (8). In the current study, it was observed that the satisfaction rates were higher in cases with cauterization in terms of the overall picture, the difference was, however, not significant and the recurrence rates did not differ between the two groups, supporting the view that the two surgical methods are not different from each other in terms of those features. Similarly, in a meta-analysis study conducted by Du et al. (9), it was detected that the cauterization and the clipping method gave similar results in terms of patient satisfaction in primary focal hyperhidrosis case.

\section{Result}

Of the interview about the treatment satisfaction with people with a diagnosis of primary focal hyperhidrosis evaluated in the study, it was seen that the rate of people who were dissatisfied with ETS treatment was $6.4 \%$. Thus, other individuals were found to be partially or significantly satisfied with the treatment. In a study in which 
hyperhidrosis patients undergoing surgical treatment were followed up, it was evaluated that $84 \%$ of the cases had an increase in the quality of life, also, $97 \%$ of the cases were satisfied with the surgical procedure (10). For this reason, it can be said that the satisfaction level reported by the cases in this study was consistent with the literature. Primary focal hyperhidrosis negatively affects the daily activities of people and can cause psychological and social problems $(2,11)$. Therefore, the data obtained from this study support the view that ETS used in the treatment of hyperhidrosis is one of the most reliable treatment methods that help to solve the social and psychological problems caused by the disease in addition to the physical symptoms (1).

According to logistic regression analysis, it has been reported that being older than 21 years of age has negatively affected the treatment results in patients who underwent ETS due to primary focal hyperhidrosis (12). Leiderman et al. (13) stated that the effect of age on patient satisfaction and quality of life in cases of hyperhidrosis treated surgically was not sufficiently examined, therefore, in their study, sweating symptoms decreased more with increased age and better results were obtained, especially in elderly individuals. Karaçam et al. (14) found that the risk and severity of compensatory hyperhidrosis increased with both age and prolongation of symptom duration. The ages of the cases evaluated in this study ranged from 12 to 44 years, and the study found that the risk for dissatisfaction with treatment increased with age. Hence, the result of this study shows that satisfaction with treatment may be affected by age characteristics. Moreover, new studies with larger samples and a wider age range can contribute to the literature by showing the effect of age characteristics of hyperhidrosis cases on treatment results.

ETS is primarily a treatment method for cases that do not respond to medical treatment and offers patients a new opportunity to recover (3). Therefore, one of the factors that significantly reduces the risk of dissatisfaction with the treatment in this study can be considered as an expected condition that the disease does not recur. In other words, failure to satisfy the treatment expectations of patients who expect recovery after the surgical procedure will be one of the factors that significantly reduce treatment satisfaction.

The recurrence rate in the study was stated as $14.9 \%$ in hyperhidrosis cases, whereas lower recurrence rates were reported in the literature. Besides, the global recurrence rate was $8.8 \%$ in cases who underwent surgery, whereas it was reported that sweating in $86.4 \%$ of the patients could continue at a compensatory degree (15). However, in a study carried out by Doğru et al. (16), recurrence rates of hyperhidrosis cases surgically treated in Turkey were detected to be $16.4 \%$ and it can be said that this rate is similar to the findings obtained from the current study. The retrospective analysis of the data and the fact that the severity of ongoing sweating symptoms was not examined in detail may affect the recurrence rate in the current study. Besides, the change in follow-up periods of 2-5 years may also be an effective factor in the recurrence rate.

In the study, although the satisfaction rates were similar between the cases who underwent cauterization and the clipping method, it was found that the cauterization method reduced the risk of dissatisfaction with the treatment in the logistic regression analysis. However, it was found that surgical methods did not increase the risk in terms of recurrence. In other words, in this study, it was found that the clipping method was a factor that reduces the satisfaction of the treatment, but the surgical methods were ineffective in increasing the risk of recurrence. It has been known that regression models in studies give more reliable results than analyzes comparing proportional data (16). Therefore, considering the results obtained from this study, it can be said that a higher satisfaction level is more likely in cases evaluated at the end of two years and treated with cauterization. Du et al. (9) and Divisi et al. (8) have reported that cauterization and clipping methods did not make a difference in terms of treatment satisfaction and that the clipping method was more disadvantageous in terms of some risks (e.g., infection). Besides, the limited number of participants in the study restricted the use of multiple regression analyzes, thus, the effect of confounding variables on the results obtained could not be analyzed. Therefore, the results of new studies that control the severity of the disease and the effects of other variables and that include multiple regression models to determine the superiority of different surgical methods used in ETS may contribute to the literature.

Reflex sweating has been one of the complications that occur due to the surgical procedure in cases of hyperhidrosis (17). In the current study, it was found that $66 \%$ of the operated cases had reflex sweating and the risk for dissatisfaction with the treatment was significantly reduced in cases without this complication. Reflex sweating has been one of the undesirable surgical complications after the operation, especially for surgeons performing ETS. In a study, it has been found that 37.5\% of the hyperhidrosis cases undergoing surgical procedures had reflex sweating (18). After ETS, it has been reported that reflex sweating developed in 46 of 247 cases and the treatment of this complication could last for two years (19). It should be, therefore, kept in mind that reflex 
sweating that develops after ETS is one of the important factors that reduce the treatment satisfaction of patients.

\section{Study Limitations}

The low number of cases is one of the important limitations of the study. Besides, the fact that multiple regression analysis cannot be used due to the limited distribution of the number of subjects according to the variables is another limitation of the study. Moreover, retrospective studies offer more limited results than prospective studies. The last limitation of the study is that patient satisfaction levels were determined by the interview method rather than a measurement tool. Despite these limitations, the presented study may contribute to the literature in evaluating the surgical methods applied in terms of patient satisfaction.

\section{Conclusion}

As a result of the study, it can be said that recurrence, reflex sweating, and satisfaction rates are similar among the cases treated with cauterization or clipping, in addition to this, increase in age, the use of clipping method, recurrence, and the presence of reflex sweating in cases of primary focal hyperhidrosis are effective factors in reducing patient satisfaction. Besides, new studies examining the effect of the clipping method on patient satisfaction may contribute to the literature.

Financial Disclosure: The author declared that this study received no financial support.

\section{References}

1. Cerfolio RJ, De Campos JR, Bryant AS, et al. The Society of Thoracic Surgeons expert consensus for the surgical treatment of hyperhidrosis. Ann Thorac Surg 2011;91:1642-8.

2. Haider A, Solish N. Focal hyperhidrosis: diagnosis and management. CMAJ 2005;172:69-75.

3. Apilioğulları B, Bilgiç Ö. Hiperhidrozis tedavisi. Genel Tip Dergisi 2014;24:79-84.

4. Nawrocki S, Cha J. The etiology, diagnosis, and management of hyperhidrosis: A comprehensive review: Therapeutic options. J Am Acad Dermatol 2019;81:669-80.

5. Santini M, Fiorelli A. Surgery for palmar hyperhidrosis: patient selection and extent of surgery. Difficult Decisions in Thoracic Surgery 2014;725-43.

6. Bagheri R, Sharifian Attar A, Haghi SZ, Salehi M, Moradpoor $\mathrm{R}$. Thoracoscopic sympathicotomy in the treatment of palmar hyperhidrosis. Asian Cardiovasc Thorac Ann 2016;24:687-91.
7. Findikcioglu A, Kilic D, Hatipoglu A. Is clipping superior to cauterization in the treatment of palmar hyperhidrosis? Thorac Cardiovasc Surg 2014;62:445-9.

8. Divisi D, Zaccagna G, Di Francescantonio W, et al. Endoscopic thoracic sympathectomy or sympathicotomy versus clipping in the surgical management of primary hyperhidrosis: a systematic review and meta-analysis. Shanghai Chest 2019;3:1-5.

9. Du X, Zhu X, Wang T, et al. Compensatory hyperhidrosis after different surgeries at the same sympathetic levels: a metaanalysis. Ann Transl Med 2018;6:203.

10. Horslen LC, Wilshire CL, Louie BE, Vallières E. Long-Term Impact of Endoscopic Thoracic Sympathectomy for Primary Palmar Hyperhidrosis. Ann Thorac Surg 2018;106:1008-12.

11. Li DC, Hulbert A, Waldbaum B, et al. Endoscopic thoracic sympathectomy for primary focal hyperhidrosis: impact on psycho-social symptomatology and psychotropic medication use. Eur J Cardiothorac Surg 2018;54:904-11.

12. Kara M, Kose S, Cayirci CE, Koksal A. Can we predict the compensatory hyperhidrosis following a thoracic sympathectomy? Indian J Thorac Cardiovasc Surg 2019;35:190-5.

13. Leiderman DBD, Milanez de Campos JR, Kauffman $P_{\text {, et }}$ al. The relation between age and outcomes of thoracic sympathectomy for hyperhidrosis: The older the better. J Thorac Cardiovasc Surg 2018;156:1748-56.

14. Karaçam V, Sanli A, Tertemiz KC, Tasdögen A. The effect of micro-invasive endoscopic thoracic sympathectomy in palmar hyperhidrosis patients on quality of life and hyperhidrosis. Turk Gogus Kalp Damar Cerrahisi Derg 2017;25:638-643.

15. Gossot D, Galetta D, Pascal A, et al. Long-term results of endoscopic thoracic sympathectomy for upper limb hyperhidrosis. Ann Thorac Surg 2003;75:1075-9.

16. Dogru MV, Sezen CB, Girgin O, et al. Is there any relationship between quality of life and the level of sympathectomy in primary palmar hyperhidrosis? Single-center experience. Gen Thorac Cardiovasc Surg 2020;68:273-9. Sur P, Chen Y, Candès EJ. The likelihood ratio test in high-dimensional logistic regression is asymptotically a rescaled chi-square. Probability Theory and Related Fields 2019;175:487-558.

17. Choi BC, Lee YC, Sim SB. Treatment of palmar hyperhidrosis by endoscopic clipping of the upper part of the T4 sympathetic ganglion. Preliminary results. Clin Auton Res 2003;1:48-51.

18. Sezen CB, Akboğa SA, Süleymanov A, Gokce A, Çelik A, Kurul iC. Is oxybutynin treatment as effective as surgery in palmar and axillary hyperhidrosis?. Haseki Tip Bulteni 2017;55:199204. 